\title{
Sales and Related Occupations
}

National Cancer Institute

\section{Source}

National Cancer Institute. Sales and Related Occupations. NCI Thesaurus. Code 697644.

A class of professional or vocational positions of employment that involve sales or related activities. 